\title{
Review Article \\ The Role of Habenula in Motivation and Reward
}

\author{
Marc Fakhoury and Sergio Domínguez López \\ Department of Neuroscience, Faculty of Medicine, University of Montreal, Montreal, QC, Canada H2V 2S9 \\ Correspondence should be addressed to Marc Fakhoury; marc_fakhoury@hotmail.com
}

Received 2 June 2014; Revised 29 July 2014; Accepted 13 August 2014; Published 26 August 2014

Academic Editor: Daniela Schulz

Copyright (C) 2014 M. Fakhoury and S. Domínguez López. This is an open access article distributed under the Creative Commons Attribution License, which permits unrestricted use, distribution, and reproduction in any medium, provided the original work is properly cited.

\begin{abstract}
Located centrally along the dorsal diencephalic system, the habenula is divided into two structures: the medial and the lateral portions. It serves as an important relay between the forebrain and several hindbrain sites. In the last few years, a huge attention has been devoted to this structure, especially the lateral habenula (LHb), which seems to play an important role in emotion, motivation, and reward. Recent studies using techniques such as electrophysiology and neuroimaging have shown that the LHb is involved in motivational control of behavior. Its dysfunction is often associated with depression, schizophrenia, and mood disorder. This review focuses on providing a neuroanatomical and behavioral overview of some of the research previously done on the LHb. First, we describe the anatomical structure of the habenula and we explain how it is involved in reward and motivation. Then, we will discuss how this structure is linked to the limbic system, to finally provide a comparison between several studies that have used electrolytic lesions.
\end{abstract}

\section{Introduction}

The study of the habenula started in the early 1980s [1], and it was known that this structure has the potential to control several regions of the midbrain. However, it was just recently that scientists discovered the influence of the habenula in motivational states, mood disorders, and several other mental problems $[2,3]$. The habenula, which is part of the epithalamus, is composed of the lateral habenula ( $\mathrm{LHb}$ ) and the medial habenula $(\mathrm{MHb})$, each having distinct functions and a unique anatomical structure. Here, we will focus on the $\mathrm{LHb}$, which is more involved with pain processing, reward, motivation, and learning $[4,5]$. In rodents, studies have shown that lesions at the level of the habenula cause cognitive impairment, attention deficits, hyperreactivity to stress, and schizophrenic-like symptoms [6]. Moreover, additional studies have linked the activity of the LHb to the regulation of serotonin (5-HT) and norepinephrine, suggesting that this structure modulates multiple regions in the brain [7-9]. It was also shown that neurons in the habenula are responsive to peripheral nociceptive stimulation, and when exposed to stress, the level of $\mathrm{c}$-fos in this structure significantly increases $[10,11]$.
The habenula receives its main afferents from the septum and stria medullaris $(\mathrm{sm})$ [8]. Furthermore, the habenula projects towards many areas in the midbrain that are involved in the release of neuromodulators and also towards dopaminergic midbrain nuclei such as the ventral tegmental area (VTA) [2, 12]. Studies utilizing lesions of the afferent projections of the LHb have demonstrated an increase in the level of anxiety in rats, with neonatal rats exhibiting a higher state of anxiety compared to adult rats [13]. Lesions of the $\mathrm{LHb}$ also induce impairment of memory and attention in rats, suggesting a connection between the LHb and cognition $[5,6,13]$. Here, we will discuss how studies using lesions and electrical self-stimulation have been able to show that the LHb is an important component of the brain reward system. We will begin by describing how the activity of $\mathrm{LHb}$ neurons can be influenced by regulatory synapses and neuromodulators and how they interact with the limbic system in the brain, to finally discuss the ability of the LHb in encoding rewarding stimuli.

\section{Anatomical Organization of the Habenula}

The primary input regions to the $\mathrm{LHb}$ are the lateral preoptic area, the ventral pallidum, the lateral hypothalamus, and 
the internal segment of the globus pallidus (GPi) [14]. The habenula is connected to the anterior diencephalon in the epiphysis peduncle. While the $\mathrm{MHb}$ receives its main afferents from the septum, the LHb is connected to the striatum and projects onto the midbrain $[9,15]$. The habenula is present across all species and is greater in size relative to the whole brain of most mammals [5]. It is a bilateral structure located above the thalamus at its posterior end and directly adjacent to the midline. Figure 1 illustrates coronal sections of the rat brain that clearly show that the habenula is a bilateral structure divided into a medial portion and a lateral portion.

Neurons in the habenula are heterogeneous in nature. In fact, studies using a Golgi staining showed that these neurons can adopt various morphologies. Neurons in the $\mathrm{MHb}$ are divided into two groups: the first group of neurons has piriform cell bodies with a mean diameter of $12 \mu \mathrm{m}$ and two to five primary dendrites, while the second group is comprised of neurons that are fusiform in shape and have around three primary dendrites that arose from cell bodies [16]. Furthermore, the phenotype of neurons of the LHb differs from the neurons in the $\mathrm{MHb}$. The $\mathrm{MHb}$ includes a dense aggregate of cholinergic neurons and neurons producing substance $\mathrm{P}$ $[16,17]$, whereas in the LHb, the majority of the neurons are glutamatergic [18]. In LHb neurons, GABAergic transmission arises from the medial globus pallidus (MGP). GABA receptors can be coupled to $G$ protein-coupled inwardlyrectifying potassium channels (GIRK) for potassium ions intake [1]. Glutamate transmission also arises from MGP and to a lesser extent from the lateral hypothalamus. It largely relies on acid receptors that can be calcium-permeable (CPAMPAR) or calcium-impermeable (CI-AMPAR). Moreover, the dopamine (DA) released from the VTA can bind to $\mathrm{D} 2 \mathrm{R}$ and $\mathrm{D} 4 \mathrm{R}$ receptors of dopaminergic cells. LHb neurons also express G-protein coupled receptors (GPCR) specific to glutamate in addition to 5 -HT2C receptors that can detect 5HT released from the dorsal raphe [1].

\section{Role of the Habenula in Reward and Its Relationship with the Limbic System}

The $\mathrm{LHb}$ is considered to be the highway to the midbrain for processing aversion and reward $[1,2]$. Studies have shown that neurons in the LHb are "reward-negative," meaning that they can get activated by stimulus associated with no reward or unpleasing events [20]. Indeed, it has been demonstrated in monkeys that the omission of an expected reward leads to a strong increase in the activity of LHb neurons, while unexpected delivery of rewards decreases LHb neuron firing [21]. It was also shown that optogenetic activation of excitatory projections towards the LHb drives aversive behaviors [1]. The habenula receives its main afferents from the septum through the sm and is part of the epithalamus $[8,22]$. The epithalamus, which is also composed of the pineal body, is connected to the basal ganglia and the limbic system. All afferents go to the LHb through the sm. However, the projections leaving the $\mathrm{LHb}$ go to the fasciculus retroflexus, which is composed of major axons that form a link between the projections of the habenula and midbrain structures.
Several other areas transmit information to the habenula from the sm, such as the lateral hypothalamus, the lateral preoptic area, the nucleus of diagonal band of Broca, and the septum. Also, the caudate transmits information to the GPi, which in turn sends projections to the LHb. Furthermore, the neurons of the $\mathrm{LHb}$ project towards many areas of the midbrain that send signals to the forebrain enabling the release of DA in the striatum $[2,12]$.

DA is a neurotransmitter that belongs to the class of catecholamines. It is synthesized from the amino acid tyrosine and is mainly produced in the substantia nigra and in the VTA [23]. A dysfunction of DA dopamine neuronal activity is associated with a variety of important diseases of the nervous system. Thus, a better understanding of the dopaminergic system will help scientists elucidate the biological and neurological mechanisms implicated in many neurodegenerative diseases. DA has received the most attention for its role in building rewarding behaviors, including behaviors that contribute to addiction and drug abuse [24, 25]. Located upstream of the mesolimbic dopaminergic pathway, the habenula is closely linked to reward because of its ability to activate dopaminergic neurons. Indeed, several studies show that the habenula has glutamatergic projections that establish their synaptic contacts with dopaminergic cells [26-28]. Moreover, the habenula is capable of communicating with dopaminergic neurons in the VTA (Figure 2 ) by activating the rostral medial tegmental nucleus (RMTg) [2, 29].

Input from the basal ganglia to the habenula, specifically the LHb, derives from the border region of the GPi, which receives inputs from the striosome subterritory of the striatum (Striatum-S) [2]. The habenula then influences DA neurons via inhibitory neurons in the RMTg. Activation of neurons in the LHb causes a decrease of the activity of dopaminergic neurons, whereas inhibition of the LHb accompanies activation of dopaminergic neurons [30]. As shown in Figure 2, motor activity is directly mediated by the substantia nigra pars reticulata $(\mathrm{SNr})$ and the $\mathrm{GPi}$, which are influenced by the innervation of the matrix subterritory of the striatum (Striatum-M), the DA neurons in the VTA, and in the substantia nigra pars compacta $(\mathrm{SNc})$.

\section{Role of the Habenula in Pain and Mood Disorders}

The habenula is a structure involved in behavioral response to pain and is also implicated with psychiatric disorders such as depression and schizophrenia [2, 31]. Several animal studies support its role in pain and analgesia [32]. It has been shown that the habenula receives information from forebrain structures and the dorsal diencephalic conduction system, which is a major site that interconnects the limbic forebrain with the midbrain [33]. The habenula also modulates the intensity of pain, aversion, and motor responses. It receives projections from the spinal cord, the limbic system, and cortical structures, while its efferent pathways are in areas of the brainstem involved in pain modulation $[5,32]$. The $\mathrm{MHb}$ projects predominantly to the interpeduncular nucleus (IPN) in the midbrain, while the LHb has projections to the VTA, 


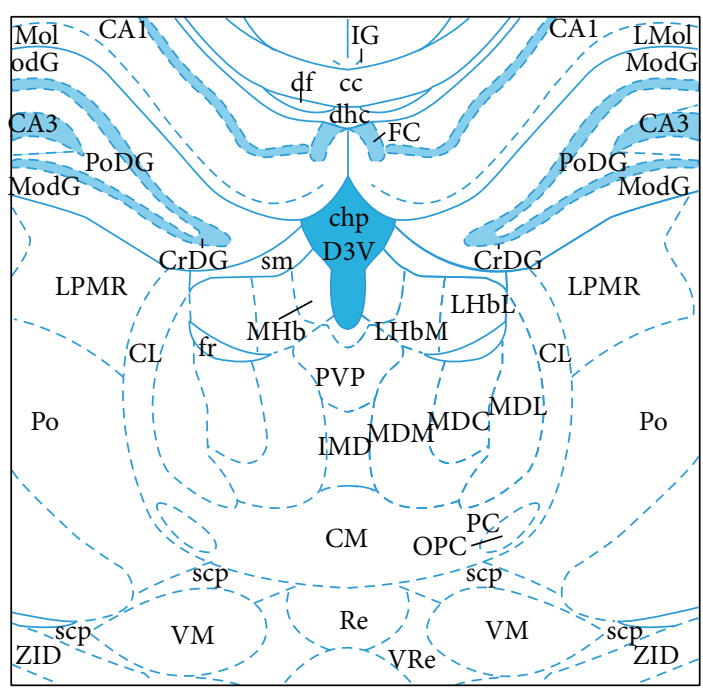

(a)

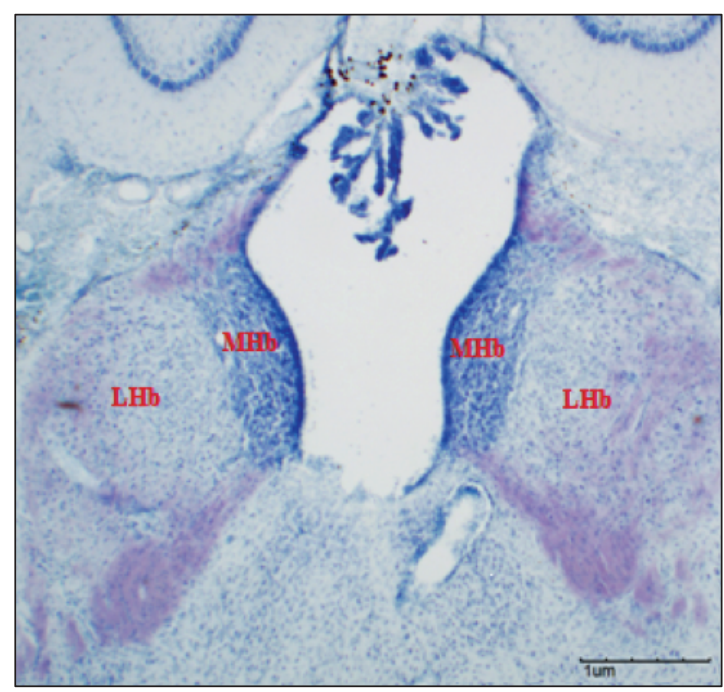

(b)

FIGURE 1: (a) Coronal section of the rat brain showing the habenula and its subdivision (taken from an atlas of the rat brain [19]). (b) A Thionin staining of a coronal section of the rat brain showing that the habenula is divided into a medial (MHb) portion and lateral (LHb) portion.

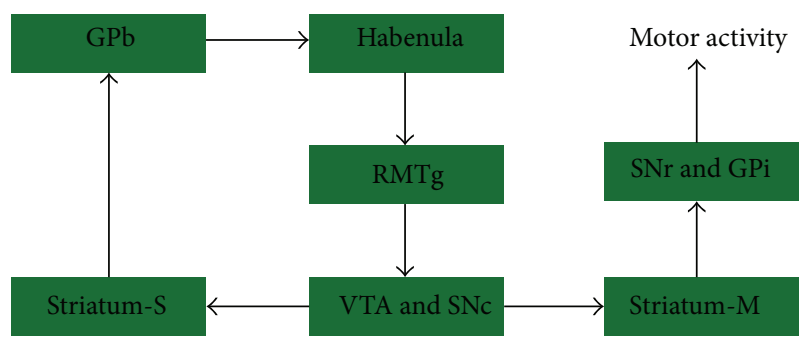

FIGURE 2: Dopamine-mediated circuit.

the dorsal raphe, and the SNc $[34,35]$. The IPN modulates cells in the periaqueductal gray and the dorsal raphe, which both play a major role in descending modulation of pain. The dorsal raphe also sends afferents directly to the spinal cord via the raphe magnus in the medulla, through serotonergic mediated pain modulatory processing [5]. Studies using electrophysiology or electrostimulation have clear indication that the habenula may be involved in pain modulation. It was shown that the $\mathrm{LHb}$ responds to noxious stimuli that may be excitatory or inhibitory [11] and that this structure is a central target for the nociceptive input at the upper brainstem level $[36,37]$. Moreover, studies using c-fos immunohistochemistry as a marker of neuronal activity show increases in neuronal stains in the LHb in experimental pain $[38,39]$. Another study utilizing diabetes-induced pain in rats showed that the pain in diabetic neuropathy significantly decreased the activation of the habenula, suggesting that the latter structure represents part of a modulatory pain system [40].

It is also well documented that the $\mathrm{LHb}$ is hyperactive in individuals with mood disorder such as depression and in animal models of this psychiatric disorder [41, 42]. Indeed, the habenula plays a crucial role in regulating behavioral responses to stress and shows increased cerebral blood flow because of its hyperactivity [43, 44]. Consistent with these data, recent studies reported a decrease in the habenula volume of unmedicated patients with bipolar disorder and major depressive disorder (MDD) [45]. Also, deep brain stimulation of the $\mathrm{LHb}$ was found to induce remission of symptoms in patients with MDD $[46,47]$.

\section{Comparison of Studies Done with Electrolytic Lesions}

Several studies have done lesions in regions of the brain in order to determine the neural circuitry involved in motivation and reward. Lesioning is a technique in which a specific region of interest in the brain is destroyed to observe any resulting changes such as altered performance on some behavioral measure. Using a 3-dimensional atlas of the brain, one can stereotaxically implant electrodes with very high accuracy to produce a lesion. Another technique commonly employed is the infusion of excitotoxic drugs into the brain, which causes death of neurons with high accuracy and efficacy [48, 49]. However, most of the studies done to examine the function of the habenula relied on lesions to this site by passing an electric current through electrodes implanted in the brain of animals [3, 50]. Following the passage of the current, the cell bodies and axons that are located at the tip of the electrode are destroyed. Destruction of neuronal cells within a confined zone should be reflected in a deterioration of the effectiveness of brain stimulation reward: when the animal receives stimulation, the reward signal gets attenuated. This technique is very useful in research and is useful to determine how eliminating the activity of specific areas in the brain can change the behavior. 
A study conducted in the University of Maryland in Baltimore has shown that the habenula can govern the attribution of incentive salience to reward predictive cues [51]. Incentive salience attribution endows a reward-predicting stimulus with the capacity to initiate goal-directed behaviour [52]. It is a type of motivation in the brain associated with an external stimuli or reward that drives the addictive behaviors. In this study, researchers used electrolytic lesions of the fasciculus retroflexus (fr), a pathway through which descending habenula efferents are conveyed. The results showed that lesioning the fr significantly increased incentive salience, suggesting that the habenula is involved in regulating the relative degree of incentive salience assigned to a given cue, which is critical for motivation and reward. Moreover, they showed that changing the activity of neurons in the LHb could result in avolition and depression-like symptoms [51]. Another study from Morissette and Boye [3] has used electrolytic lesions aimed at the $\mathrm{LHb}$ in order to demonstrate the role that this structure plays in motivation and reward. Following the implantation of stimulation and lesioning electrodes, the rats were trained at several currents and were put in a cage with a lever that could self-administer the stimulation. Each time the rat pressed the lever, the rewarding stimulation was transmitted to its brain. Behavioral curves correlating the number of presses with the frequency of the stimulation were then generated. From these curves, the number of presses required to obtain half-maximal responding, known as the reward threshold, was calculated. It was shown that lesioning the LHb significantly increases the reward threshold and alters the behavior of the rats. The increase in reward threshold was the result of destruction of habenular neurons that seem to be necessary in transmitting the reward signal.

By regulating the 5-HT and DA system, the habenula may also has an implication in Parkinson's disease [2, 12, 50]. Patients with Parkinson's disease suffer from motor disturbances, which result from the death of DA-generating cells in the substantia nigra. To test whether the habenula is implicated in this disease, scientists have monitored the behavior of rats after functionally suppressing the LHb activity [50]. The results of their study showed that hyperactivity in the $\mathrm{LHb}$ downregulates the serotonergic system, thus resulting in depressive symptoms in patients with Parkinson's disease. However, this process can be partially reversed by DA replacement therapy and habenula lesion. These findings provide a link between Parkinson's disease and depression and open up avenues for new therapeutic interventions.

\section{Conclusion}

Evidence collected in the last 20 years illustrated the potential of the $\mathrm{LHb}$ in controlling the midbrain, but not much was known of the anatomy of this structure and its synaptic organization. Only recently it is starting to become clear that this structure participates in the encoding of aversive and rewarding stimuli, influences motivational states, and contributes to pathologies such as mood disorders and addiction [1]. Such discoveries have the potential to rapidly advance our knowledge and can open up avenues for therapeutic interventions. Indeed, by understanding the function of the habenula and its implication in brain reward circuits, scientists could find new molecular and genetic targets that could potentially aid in the treatment of several neurological diseases. As presented in this review, the habenula is strongly connected to the limbic system and plays an important role in regulating directed behavior. It receives its main afferents from the septum through the sm and projects towards several areas of the midbrain involved in the release of neuromodulators. The presence of dopaminergic projections that innervate the $\mathrm{LHb}$ has also been described, suggesting that the habenula could be implicated in psychological disorders such as depression, addiction, and drug abuse. Finally, most of the understanding of the function of the LHb so far, has been done by observing how the behavior of subjects was altered after lesioning this structure. Several studies found that the LHb plays a critical part of the reward system and that a dysfunction of the LHb is often associated with psychiatric disorders $[36,37,50]$. The studies presented here discuss several pathways in which the habenula is involved and describe several neurotransmitters that act in the LHb, including amino acids such as glutamate and GABA. However, evidence suggests that other potentially relevant molecules may also act in the $\mathrm{LHb}$, including orexins, acetylcholine, and vasopressin $[1,42]$. Several studies have shown that the habenula is implicated in the release of neuromodulators such as DA and 5-HT, but the big challenge now is to determine the downstream implications of such changes on reward and motivation. There are several studies that still need to be done on the habenula, and what will be learned in the next few years will allow us to better understand how regulatory systems control motivated behavior and may offer a window to further develop therapeutic strategies.

\section{Conflict of Interests}

The authors declare no conflict of financial interests.

\section{References}

[1] F. J. Meye, S. Lecca, K. Valentinova, and M. Mameli, "Synaptic and cellular profile of neurons in the lateral habenula," Frontiers in Human Neuroscience, vol. 7, p. 860, 2013.

[2] O. Hikosaka, "The habenula: from stress evasion to value-based decision-making," Nature Reviews Neuroscience, vol. 11, no. 7, pp. 503-513, 2010.

[3] M. Morissette and S. M. Boye, "Electrolytic lesions of the habenula attenuate brain stimulation reward," Behavioural Brain Research, vol. 187, no. 1, pp. 17-26, 2008.

[4] K. H. Andres, M. V. During, and R. W. Veh, "Subnuclear organization of the rat habenular complexes," The Journal of Comparative Neurology, vol. 407, no. 1, pp. 130-150, 1999.

[5] L. Shelton, L. Becerra, and D. Borsook, "Unmasking the mysteries of the habenula in pain and analgesia," Progress in Neurobiology, vol. 96, no. 2, pp. 208-219, 2012.

[6] L. Lecourtier, H. C. Neijt, and P. H. Kelly, "Habenula lesions cause impaired cognitive performance in rats: implications for schizophrenia," European Journal of Neuroscience, vol. 19, no. 9, pp. 2551-2560, 2004. 
[7] J. Amat, P. D. Sparks, P. Matus-Amat, J. Griggs, L. R. Watkins, and S. F. Maier, "The role of the habenular complex in the elevation of dorsal raphe nucleus serotonin and the changes in the behavioral responses produced by uncontrollable stress," Brain Research, vol. 917, no. 1, pp. 118-126, 2001.

[8] M. Herkenham and W. J. H. Nauta, "Efferent connections of the habenular nuclei in the rat," Journal of Comparative Neurology, vol. 187, no. 1, pp. 19-47, 1979.

[9] K. M. Velasquez, D. L. Molfese, and R. Salas, "The role of the habenula in drug addiction," Frontiers in Human Neuroscience, vol. 8, article 174, 2014.

[10] D. Wirtschafter, K. E. Asin, and M. R. Pitzer, "Dopamine agonists and stress produce different patterns of Fos-like immunoreactivity in the lateral habenula," Brain Research, vol. 633, no. 1-2, pp. 21-26, 1994.

[11] D. M. Gao, D. Huffman, and A. L. Benabid, "Simultaneous recording of spontaneous activities and nociceptive responses from neurons in the Pars compacta of Substantia nigra and in the lateral habenula," European Journal of Neuroscience, vol. 8, no. 7, pp. 1474-1478, 1996.

[12] P. Baldwin, R. Alanis, and R. Salas, "The role of the Habenula in nicotine addiction," Journal of Addiction Research \& Therapy, vol. S1, article 002, 2001.

[13] C. A. Murphy, A. M. DiCamillo, F. Haun, and M. Murray, "Lesion of the habenular efferent pathway produces anxiety and locomotor hyperactivity in rats: a comparison of the effects of neonatal and adult lesions," Behavioural Brain Research, vol. 81, no. 1-2, pp. 43-52, 1996.

[14] S. Geisler and M. Trimble, "The lateral habenula: no longer neglected," CNS Spectrums, vol. 13, no. 6, pp. 484-489, 2008.

[15] O. Hikosaka, S. R. Sesack, L. Lecourtier, and P. D. Shepard, "Habenula: crossroad between the basal ganglia and the limbic system," Journal of Neuroscience, vol. 28, no. 46, pp. 11825-11829, 2008.

[16] N. Iwahori, "A Golgi study on the habenular nucleus of the cat," Journal of Comparative Neurology, vol. 171, no. 3, pp. 319-344, 1977.

[17] A. Claudio Cuello, P. C. Emson, G. Paxinos, and T. Jessell, "Substance $\mathrm{P}$ containing and cholinergic projections from the habenula," Brain Research, vol. 149, no. 2, pp. 413-429, 1978.

[18] K. Brinschwitz, A. Dittgen, V. I. Madai, R. Lommel, S. Geisler, and R. W. Veh, "Glutamatergic axons from the lateral habenula mainly terminate on GABAergic neurons of the ventral midbrain," Neuroscience, vol. 168, no. 2, pp. 463-476, 2010.

[19] G. Paxinos and C. Watson, The Rat Brain in Stereotaxic Coordinates-The New Coronal Set, Academic Press, 6th edition, 2007.

[20] S. Hong, T. C. Jhou, M. Smith, K. S. Saleem, and O. Hikosaka, "Negative reward signals from the lateral habenula to dopamine neurons are mediated by rostromedial tegmental nucleus in primates," The Journal of Neuroscience, vol. 31, no. 32, pp. 1145711471, 2011.

[21] M. Matsumoto and O. Hikosaka, "Representation of negative motivational value in the primate lateral habenula," Nature Neuroscience, vol. 12, no. 1, pp. 77-84, 2009.

[22] L. W. Swanson and W. M. Cowan, "The connections of the septal region in the rat," Journal of Comparative Neurology, vol. 186, no. 4, pp. 621-655, 1979.

[23] J. H. Fallon and R. Y. Moore, "Catecholamine innervation of the basal forebrain. IV. Topography of the dopamine projection to the basal forebrain and neostriatum," Journal of Comparative Neurology, vol. 180, no. 3, pp. 545-572, 1978.
[24] R. A. Wise, "Roles for nigrostriatal-not just mesocorticolimbic-dopamine in reward and addiction," Trends in Neurosciences, vol. 32, no. 10, pp. 517-524, 2009.

[25] S. Ikemoto, "Brain reward circuitry beyond the mesolimbic dopamine system: a neurobiological theory," Neuroscience \& Biobehavioral Reviews, vol. 35, pp. 129-150, 2010.

[26] N. Omelchenko, R. Bell, and S. R. Sesack, "Lateral habenula projections to dopamine and GABA neurons in the rat ventral tegmental area," European Journal of Neuroscience, vol. 30, no. 7, pp. 1239-1250, 2009.

[27] J. J. Balcita-Pedicino, N. Omelchenko, R. Bell, and S. R. Sesack, "Sesack inhibitory influence of the lateral habenula on midbrain dopamine cells: ultrastructural evidence for indirect mediation via the rostromedial mesopontine tegmental nucleus," Journal of Comparative Neurology, vol. 519, pp. 1143-1164, 2011.

[28] C. H. Good, H. Wang, Y. H. Chen, C. A. Mejias-Aponte, A. F. Hoffman, and C. R. Lupica, "Dopamine D4 receptor excitation of lateral habenula neurons via multiple cellular mechanisms," The Journal of Neuroscience, vol. 33, no. 43, pp. 16853-16864, 2013.

[29] T. C. Jhou, S. Geisler, M. Marinelli, B. A. Degarmo, and D. S. Zahm, "The mesopontine rostromedial tegmental nucleus: a structure targeted by the lateral habenula that projects to the ventral tegmental area of Tsai and substantia nigra compacta," Journal of Comparative Neurology, vol. 513, no. 6, pp. 566-596, 2009.

[30] M. Matsumoto and O. Hikosaka, "Lateral habenula as a source of negative reward signals in dopamine neurons," Nature, vol. 447, no. 7148, pp. 1111-1115, 2007.

[31] G. Ellison, "Stimulant-induced psychosis, the dopamine theory of schizophrenia, and the habenula," Brain Research Reviews, vol. 19, no. 2, pp. 223-239, 1994.

[32] L. Shelton, G. Pendse, N. Maleki et al., "Mapping pain activation and connectivity of the human habenula," Journal of Neurophysiology, vol. 107, no. 10, pp. 2633-2648, 2012.

[33] I. H. Bianco and S. W. Wilson, "The habenular nuclei: A conserved asymmetric relay station in the vertebrate brain," Philosophical Transactions of the Royal Society B: Biological Sciences, vol. 364, no. 1519, pp. 1005-1020, 2009.

[34] G. R. Christoph, R. J. Leonzio, and K. S. Wilcox, "Stimulation of the lateral habenula inhibits dopamine-containing neurons in the substantia nigra and ventral tegmental area of the rabbit," Journal of Neuroscience, vol. 6, no. 3, pp. 613-619, 1986.

[35] C. Sego, L. Gon $\tau$ alves, L. Lima, IC. Furigo, J. Donato Jr., and M. Metzger, "Lateral habenula and the rostromedial tegmental nucleus innervate neurochemically distinct subdivisions of the dorsal raphe nucleus in the rat," Journal of Comparative Neurology, vol. 522, no. 7, pp. 1454-1484, 2014.

[36] A. L. Benabid and L. Jeaugey, "Cells of the rat lateral habenula respond to high-threshold somatosensory inputs," Neuroscience Letters, vol. 96, no. 3, pp. 289-294, 1989.

[37] M. J. Millan, "The induction of pain: an integrative review," Progress in Neurobiology, vol. 57, no. 1, pp. 1-164, 1999.

[38] W. J. Smith, J. Stewart, and J. G. Pfaus, “Tail pinch induces fos immunoreactivity within several regions of the male rat brain: effects of age," Physiology and Behavior, vol. 61, no. 5, pp. 717$723,1997$.

[39] M. Lehner, E. Taracha, A. Skórzewska et al., "Sensitivity to pain and c-Fos expression in brain structures in rats," Neuroscience Letters, vol. 370, no. 1, pp. 74-79, 2004. 
[40] P. E. Paulson, J. W. Wiley, and T. J. Morrow, "Concurrent activation of the somatosensory forebrain and deactivation of periaqueductal gray associated with diabetes-induced neuropathic pain," Experimental Neurology, vol. 208, no. 2, pp. 305313, 2007.

[41] L. Welberg, "Psychiatric disorders: reining in the habenula?" Nature Reviews Neuroscience, vol. 14, no. 10, pp. 668-669, 2013.

[42] J. Shumake, E. Edwards, and F. Gonzalez-Lima, "Opposite metabolic changes in the habenula and ventral tegmental area of a genetic model of helpless behavior," Brain Research, vol. 963, no. 1-2, pp. 274-281, 2003.

[43] J. B. Savitz, O. Bonne, A. C. Nugent et al., "Habenula volume in post-traumatic stress disorder measured with high-resolution MRI," Biology of Mood \& Anxiety Disorders, vol. 1, no. 1, article 7, 2011.

[44] J. P. Roiser, J. Levy, S. J. Fromm et al., “The effects of tryptophan depletion on neural responses to emotional words in remitted depression," Biological Psychiatry, vol. 66, no. 5, pp. 441-450, 2009.

[45] J. B. Savitz, A. C. Nugent, W. Bogers et al., "Habenula volume in bipolar disorder and major depressive disorder: a highresolution magnetic resonance imaging study," Biological Psychiatry, vol. 69, no. 4, pp. 336-343, 2011.

[46] A. Sartorius, K. L. Kiening, P. Kirsch et al., "Remission of major depression under deep brain stimulation of the lateral habenula in a therapy-refractory patient," Biological Psychiatry, vol. 67, no. 2, pp. e9-el1, 2010.

[47] H. Meng, Y. Wang, M. Huang, W. Lin, S. Wang, and B. Zhang, "Chronic deep brain stimulation of the lateral habenula nucleus in a rat model of depression," Brain Research, vol. 1422, pp. 3238, 2011.

[48] R. B. Whitelaw, A. Markou, T. W. Robbins, and B. J. Everitt, "Excitotoxic lesions of the basolateral amygdala impair the acquisition of cocaine-seeking behaviour under a second-order schedule of reinforcememt," Psychopharmacology, vol. 127, no. 3, pp. 213-224, 1996.

[49] E. D. Kirby, K. Jensen, K. A. Goosens, and D. Kaufer, "Stereotaxic surgery for excitotoxic lesion of specific brain areas in the adult rat," Journal of Visualized Experiment, no. 65, Article ID e4079, 2012.

[50] D. Sourani, R. Eitan, N. Gordon, and G. Goelman, “The habenula couples the dopaminergic and the serotonergic systems: application to depression in Parkinson's disease," European Journal of Neuroscience, vol. 36, no. 6, pp. 2822-2829, 2012.

[51] L. Carey, D. Danna Paul, and I. Greg, "The habenula governs the attribution of incentive salience to reward predictive cues," Frontiers in Human Neuroscience, vol. 7, article 781, 2013.

[52] R. A. Rescorla and R. L. Solomon, "Two-process learning theory: relationships between Pavlovian conditioning and instrumental learning," Psychological Review, vol. 74, no. 3, pp. 151-182, 1967. 

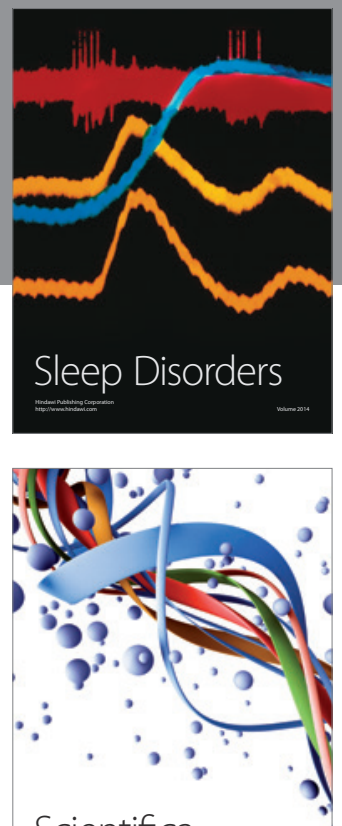

Scientifica
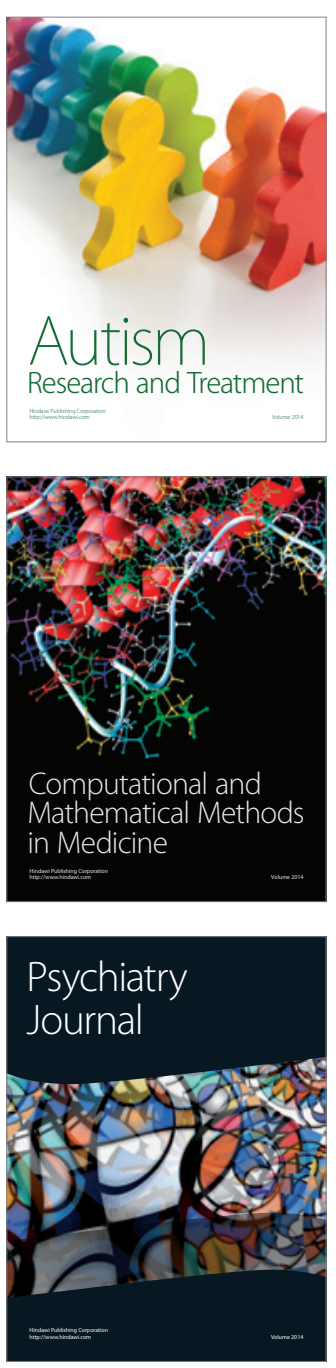
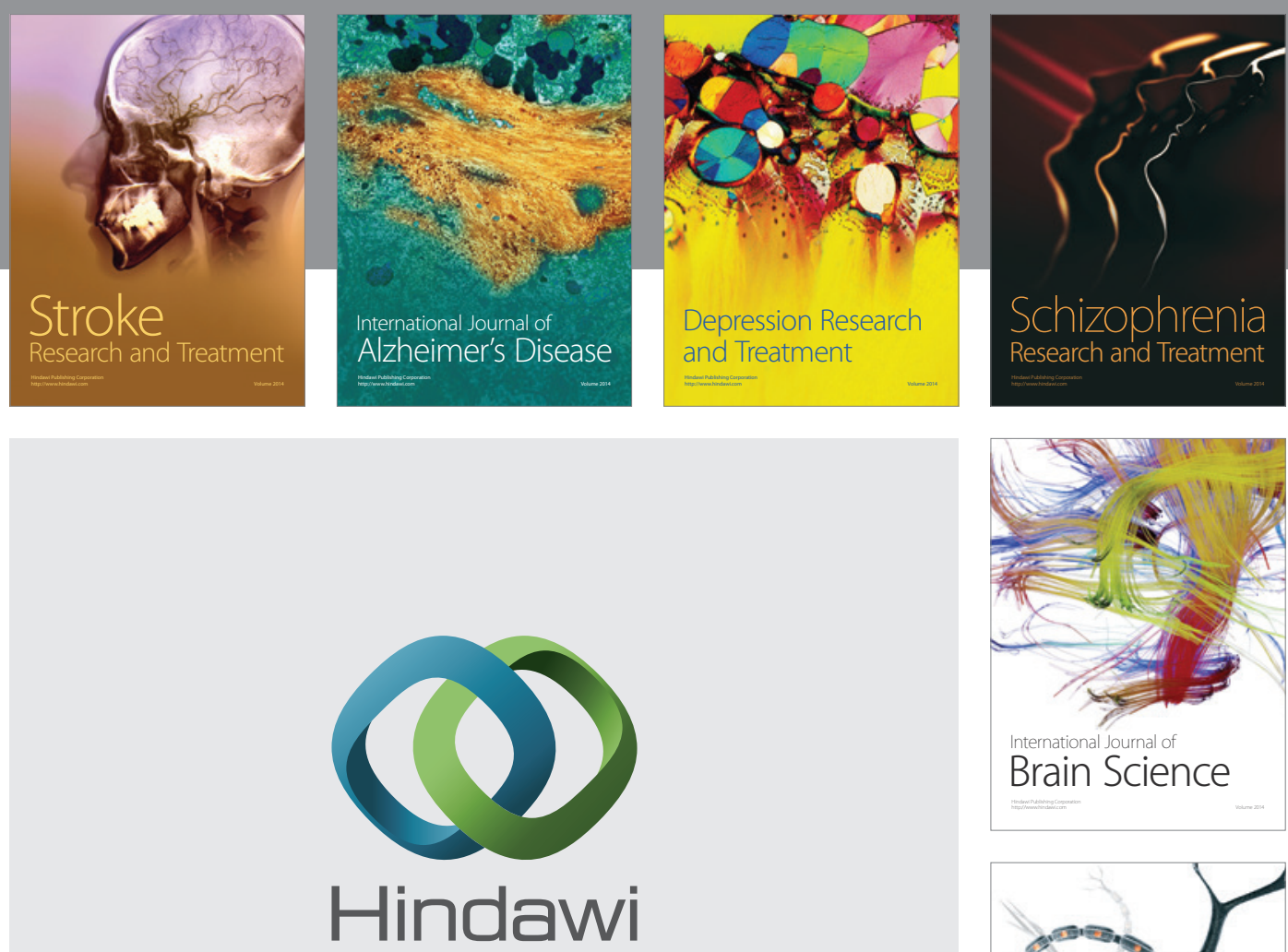

Submit your manuscripts at

http://www.hindawi.com
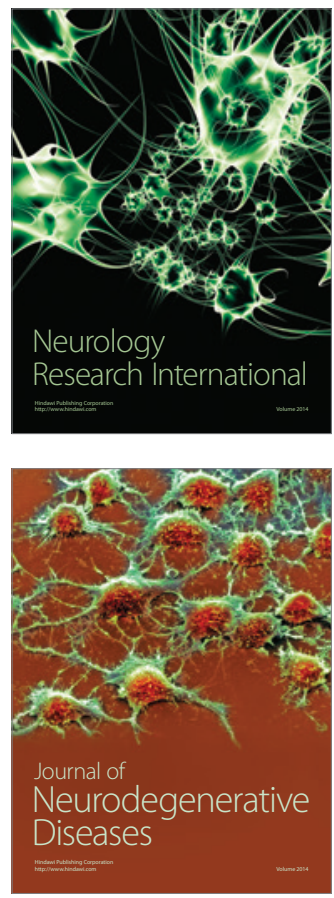

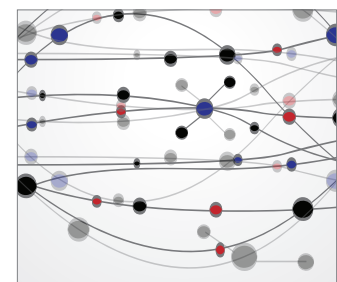

The Scientific World Journal
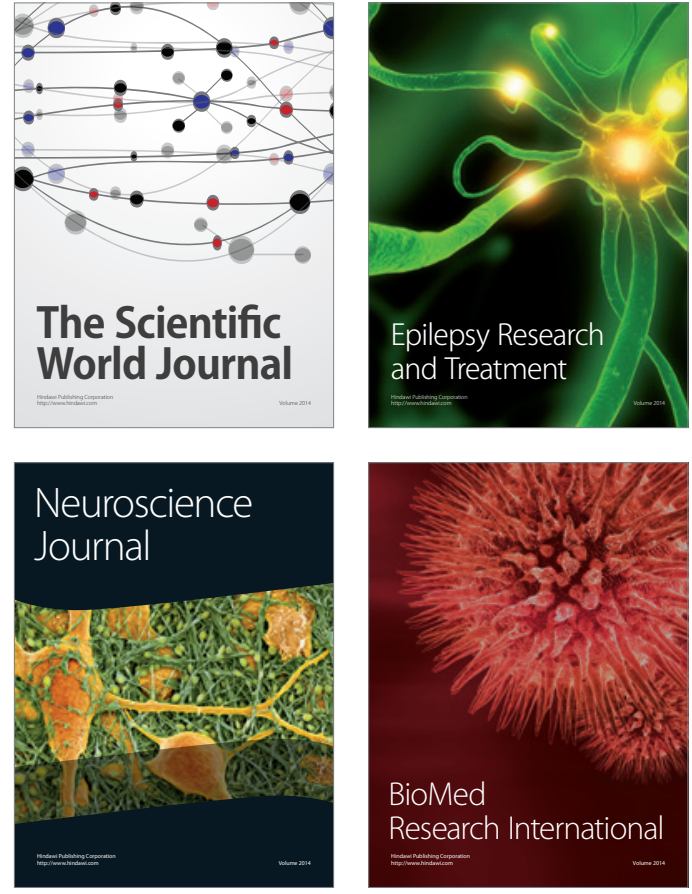

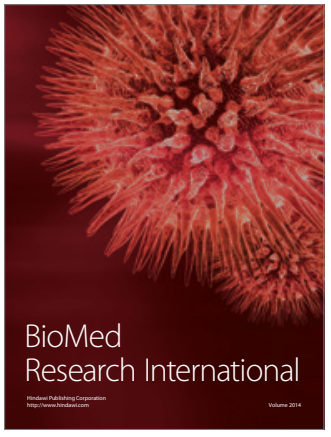

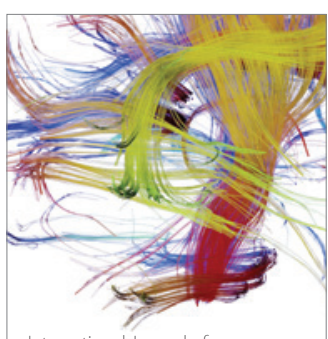

Brain Science

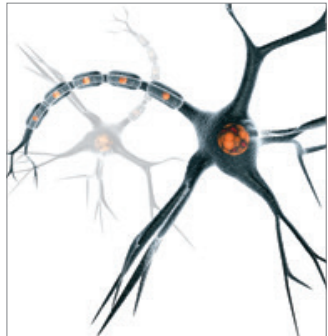

Neural Plasticity
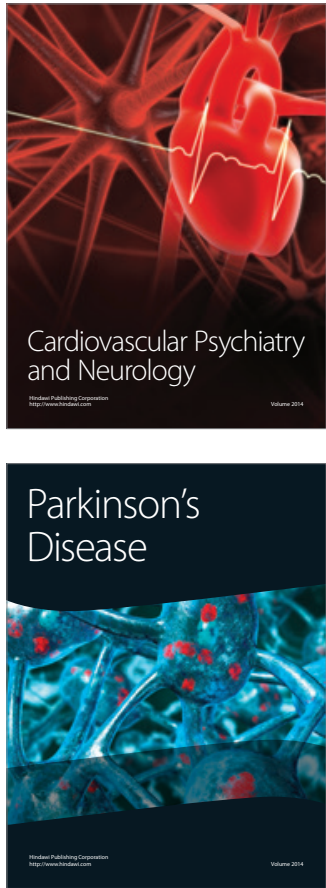